\title{
TENSIONES Y NUDOS CRÍTICOS EN LA IMPLEMENTACIÓN DE LA(S) POLÍTICA(S) DE CONVIVENCIA ESCOLAR EN CHILE ${ }^{1}$
}

\author{
Verónica López $z^{2}$ \\ Lorena Ramírez ${ }^{3}$ \\ René Valdés ${ }^{4}$ \\ Paula Ascorra ${ }^{5}$ \\ Claudia Carrasco-Aguilar 6
}

RESUMEN

Durante los últimos 15 años, Chile ha diseñado e implementado diversas leyes y políticas educativas orientadas a mejorar, promover y desarrollar la convivencia escolar y a disminuir los niveles de violencia escolar. Sin embargo, se han identificado tensiones paradigmáticas en los fundamentos de estos instrumentos, que crean un escenario ambivalente para la implementación. El objetivo de este estudio fue identificar y analizar las tensiones y nudos críticos reportados por los actores que participan del diseño e implementación de las políticas educativas en convivencia escolar. A través de una metodología cualitativa, se realizaron diez entrevistas activas-reflexivas a informantes clave de tres organismos que componen la nueva arquitectura del sistema educativo chileno (Agencia de Calidad, Ministerio de Educación y Superintendencia de Educación). El análisis de contenido temático reveló que los participantes identifican tensiones y nudos críticos a nivel conceptual (qué es la convivencia escolar), metodológico (cómo evaluarla) y procedimental (cómo entregar apoyos). Se discuten estos hallazgos desde la perspectiva de las ciencias de la implementación.

Palabras clave: convivencia escolar, implementación, política educativa, sistema de aseguramiento de la calidad, violencia escolar.

1 Este estudio fue financiado por el proyecto Fondecyt 1140960 "Políticas y prácticas de apoyo a la convivencia escolar en ambientes punitivos, segregados y de pruebas estandarizadas con altas consecuencias: el caso de Chile". Agradecimientos a Fondecyt y al proyecto CIE16009 del Programa PIA de Conicyt.

2 Escuela de Psicología y Centro de Investigación para la Educación Inclusiva, Pontificia Universidad Católica de Valparaíso, Valparaíso, Chile. Contacto: veronica.lopez@pucv.cl

3 Escuela de Psicología y Centro de Investigación para la Educación Inclusiva, Pontificia Universidad Católica de Valparaíso, Valparaíso, Chile. Contacto: lorena.ramirez@pucv.cl

4 Escuela de Pedagogía, Pontificia Universidad Católica de Valparaíso, Valparaíso, Chile. Contacto: rene.valdes@pucv.cl

5 Escuela de Psicología y Centro de Investigación para la Educación Inclusiva, Pontificia Universidad Católica de Valparaíso, Valparaíso, Chile. Contacto: paula.ascorra@pucv.cl

6 Departamento de Psicología, Universidad de Playa Ancha, Valparaíso, Chile. Contacto: claudia.carrasco@upla.cl 


\title{
TENSIONS AND CRITICAL ASPECTS OF THE IMPLEMENTATION OF SCHOOL CLIMATE POLICIES, IN CHILE
}

\begin{abstract}
During the last 15 years, Chile has designed and implemented various laws and education policies aimed at improving, promoting and developing school climate and reducing levels of school violence. However, paradigmatic tensions have been identified in the foundations of these policies and laws, which create an ambivalent scenario for their implementation. The purpose of this study was to identify and analyze the tensions and critical aspects identified by the actors involved in the design and implementation of education policies on school climate. Through a qualitative methodology, ten active-reflexive interviews were carried out with key informants from three of the different organisms that conform the new structure of Chile's educational system (Quality Agency, Ministry of Education and Superintendence of Education). Thematic content analysis revealed that the participants identified critical tensions and aspects at the conceptual level (what is school climate), methodological level (how to evaluate it), and procedural level (how to improve it). These findings are discussed from the perspective of implementation science.
\end{abstract}

Keywords: education policy, implementation, education quality assurance system, school climate, school violence.

\section{Introducción}

Durante los últimos 15 años, Chile ha diseñado e implementado diversas leyes y políticas educativas orientadas a mejorar, promover y desarrollar la convivencia escolar. Se han promulgado tres leyes directamente vinculadas con el tema de la violencia y la convivencia escolar; además de una variedad de políticas educativas, reformulaciones de la política y circulares e instructivos de implementación de la política en convivencia escolar. Desde el marco de las ciencias de implementación, es importante conocer cómo se han traducido las políticas que abordan la convivencia y violencia en las escuelas. En este artículo se abordan las tensiones y nudos críticos en la implementación de las políticas relacionadas con la convivencia escolar y/o violencia escolar, desde el punto de vista de quienes han sido encargados de implementarla a nivel central. 
98 TENSIONES Y NUDOS CRÍTICOS EN LA IMPLEMENTACIÓN DE LA(S) POLÍTICA(S) DE CONVIVENCIA ESCOLAR EN CHILE - V.López, L. Ramírez, R. Valdés, P. Ascorra y C. CarrascoAguilar

\section{Las ciencias de la implementación y la experiencia de implementadores}

El marco conceptual que guía esta investigación es el de las ciencias de la implementación. Las ciencias de la implementación (implementation sciences) han sido popularizadas en el ámbito científico durante los últimos 10 años y se refieren al estudio de métodos necesarios para llevar la evidencia y conocimiento científico a la práctica (Eccles \& Mittman, 2006; Smith \& Polaha, 2017; Thomas et al., 2016). Es por lo anterior que las ciencias de la implementación también han adquirido relevancia en otras disciplinas tales como la psicología, el marketing y las ciencias políticas (Foy et al., 2015; Rabin et al., 2012).

Puntualmente, las ciencias de la implementación ofrecen un método sistemático que puede contribuir a la instalación y evaluación del éxito o fracaso de planes, programas y políticas públicas (Tabak, Khoong, Chambers \& Brownson, 2012; Thomas et al., 2016). Lo anterior, con el objetivo de mejorar la calidad, eficiencia (en tiempo y recursos) y efectividad de ellos (Eccles \& Mittman, 2006; Smith $\&$ Polaha, 2017). De esta manera, las ciencias de la implementación emergen como una aproximación útil para aquellos que deseen maximizar el éxito de los programas o políticas públicas por implementar (Smith \& Polaha, 2017; Tabak et al., 2012).

Debido a que las ciencias de la implementación han sido abordadas desde distintos ámbitos de acción, es que se han desarrollado variados modelos que abarcan la diseminación e implementación desde distintos niveles. Tabak et al. (2012) han sistematizado más de 60 de estos modelos desde una mirada social ecológica que aborda el nivel individual, organizacional, comunitario, de políticas públicas y de sistema. Uno de ellos es el modelo de "vías para probar políticas informadas" propuesto por Bowen y Zwi (2005), que propone una toma de decisión política que se basa en la información y en las prácticas implementadas (Bowen $\&$ Zwi, 2005; Tabak et al., 2012). Considerando los factores del contexto político y rápidamente cambiante en el cual se inserta la toma de decisiones e implementación de políticas públicas, Bowen y Zwi 
(2005) identificaron diferentes evidencias para considerar el alcance e impacto de las políticas públicas, en tres niveles:

- nivel individual, evaluado a través del liderazgo; habilidades y conocimiento; recursos; organización y redes;

- nivel organizacional, evaluado a través de políticas, procesos y procedimientos; redes; distribución de recursos; liderazgo; conocimiento y habilidades; y

- nivel de sistema, evaluado a través de políticas; economía, ideología y valores.

Consideramos que un modelo integral como este puede proporcionar evidencia fundamental para la creación e implementación efectiva de políticas públicas contextualizadas. Asimismo, contribuye a la comprensión de cómo los usuarios e interesados clave las reciben, adoptan y adaptan, además de las fuerzas que constriñen o facilitan la implementación de las mismas. Finalmente, da cuenta de los valores e intereses que influirán en la percepción y respuesta a las políticas (Bowen \& Zwi, 2005).

Lo anterior resulta relevante debido a que, en Chile, muchas políticas públicas han sido implementadas de manera unilateral o con bajos niveles de participación ciudadana, afectando su pertinencia y contextualización (Astete y Vaccari, 2017). En materia de educación, desde la década de 1990 y con el regreso a la democracia, la participación de la sociedad civil y especialmente la del profesorado en el diseño de políticas educativas ha sido de carácter más bien consultivo (Leiras, 2007; Soto Guzmán, 2006; Tello, 2013; Tenorio, 2011), lo que ha contribuido a una crisis de legitimidad de la acción política y de la participación del profesorado (Cornejo y Reyes, 2008; Raczynski, Muñoz, Weinstein y Pascual, 2013).

En este artículo nos centramos en el nivel organizacional (Bowen \& Zwi, 2005). Considerando que las "vías para probar políticas informadas" son inusuales en el contexto educativo chileno, la investigación del nivel organizacional en la implementación, adaptación y traducción de la política pública podría aportar información valiosa, debido a que este nivel puede ser considerado 
100 TENSIONES Y NUDOS CRÍTICOS EN LA IMPLEMENTACIÓN DE LA(S) POLÍTICA(S) DE CONVIVENCIA ESCOLAR EN CHILE - V.López, L. Ramírez, R. Valdés, P. Ascorra y C. CarrascoAguilar

como el articulador entre el diseño centralizado de la política y las maneras en que estas son implementadas por las escuelas. Desde este punto de vista, interesa relevar la experiencia de quienes implementan las políticas de convivencia escolar, como una vía para probar política informada, es decir, como un mecanismo para informar a la propia política pública.

\section{La(s) política(s) de convivencia escolar en Chile}

El año 2002 el Ministerio de Educación de Chile, Mineduc, formuló la primera Política de Convivencia Escolar, la que explicita la relevancia de una formación integral, donde el desarrollo intelectual, ético y socioafectivo ocupó un lugar central y se realizó a través de los objetivos transversales de educación. El año 2011 se actualizó la política (Mineduc, 2011). Esta adaptación comprendió la convivencia desde una perspectiva amplia donde esta no significaba solamente la reducción de la violencia, sino principalmente la interrelación entre las personas, el respeto y la solidaridad. Finalmente, el año 2015 se publicó la Nueva Política Nacional de Convivencia Escolar 2015-2018 (Mineduc, 2015) que integró en el título los años de duración de la misma, anticipando una nueva actualización al año 2019. Esta política se enmarcó explícitamente en la nueva Reforma educacional, cuyo objetivo es avanzar hacia una educación inclusiva y de calidad en todos los establecimientos escolares, haciendo explícito un sentido formativo, de derecho, de género, de gestión territorial, y participativo.

Los antecedentes anteriores dan cuenta de los cambios de la política educativa en materia de convivencia escolar emanada desde el poder ejecutivo, particularmente desde el Mineduc, liderado por la Unidad de Transversalidad de dicha cartera. No obstante, y en forma paralela y relativamente independiente, el poder legislativo promovió y luego promulgó una ley de violencia escolar (Ley $\mathrm{N}^{\circ}$ 20.536 del año 2011, de aquí en adelante LVE). La LVE tuvo como antecedente el proyecto de ley impulsado por los senadores Carlos Cantero, Andrés Chadwick, Ricardo Lagos Weber, Ignacio Walker y Andrés Allamand. La comisión a cargo contó con la asistencia y colaboración del ministro de Educación, el subsecretario de 
Educación, la asesora jurídica del Mineduc, el jefe de la División Jurídica del Mineduc, y diversas autoridades del ámbito académico nacional y de organizaciones internacionales (Comisión de Educación, Deportes y Recreación, 2011). La LVE exigió a las comunidades escolares "hacerse responsables" de prevenir las situaciones de acoso escolar y promover la sana convivencia escolar, y para lo cual creó un nuevo cargo al interior de las escuelas, el de encargado de convivencia escolar. La LVE estableció, además, sanciones pecuniarias (multas) para los establecimientos escolares que no logran entregar evidencias del debido proceso en el abordaje de situaciones de violencia escolar.

Magendzo, Toledo y Gutiérrez (2013) identificaron dos paradigmas en tensión que sustentan la LVE. Uno es el de control y sanción, y el otro es el de convivencia democrática. Mientras que el primero aborda principalmente la inseguridad ciudadana en el ámbito escolar, el segundo se centra en el bienestar y la construcción de ciudadanía. En el primero, es la violencia escolar el objeto de intervención, y el castigo y sanción su forma de abordaje y gestión de las interacciones en la escuela; por el contrario, en el segundo, lo que se enfatiza son las medidas formativas y las sanciones reparadoras las modalidades de gestión de las interacciones.

El análisis crítico de la LVE realizado por Carrasco, López y Estay (2012) coincide con estos planteamientos. Los autores identificaron también dos paradigmas en tensión: uno de acción punitiva, más próximo a la lógica penal, que busca identificar y castigar a los agresores y a las escuelas por medio de multas y amenazas de cierre; y el otro, de acciones formativas, próximas a una lógica pedagógica, que busca resolver los conflictos mediante el diálogo y la reparación (Hevia, 2010). Del análisis concluyeron que dicha ley presentaba un carácter hibrido, pues contenía en su prescripción tanto elementos de punición (sanciones mediante multas a las escuelas) como de formación (designación de encargado de convivencia escolar; atribución al consejo escolar de las medidas de prevención y formación en las escuelas que reciben subvención estatal; y de un equipo de convivencia en las escuelas particulares pagadas). 
102 TENSIONES Y NUDOS CRÍTICOS EN LA IMPLEMENTACIÓN DE LA(S) POLÍTICA(S) DE CONVIVENCIA ESCOLAR EN CHILE - V.López, L. Ramírez, R. Valdés, P. Ascorra y C. CarrascoAguilar

Considerando la diversidad de posiciones discursivas y enfoques que sostienen los diferentes documentos legales que interpelan a las escuelas a trabajar y mejorar la convivencia escolar, en este artículo sostenemos que, aun cuando oficialmente exista una única política oficial de convivencia escolar — la del Ministerio de Educación (2015)—, performativamente la escuela se ve interpelada a actuar frente a varias y diversas políticas de convivencia escolar, que le demandan acciones, estrategias e instrumentos distintos.

\section{La nueva arquitectura chilena y los desafíos para la convivencia escolar}

A partir del año 2011, y tras la Ley de Aseguramiento de la Calidad de la Educación (Ley No 20.529), se crearon dos órganos independientes de gobierno, ambos ya funcionando. Por una parte, la Superintendencia de Educación Escolar, encargada de fiscalizar a las escuelas, entre otros asuntos, en materia de reglamentación de la convivencia escolar. Por otra, la Agencia de Calidad de la Educación, encargada de evaluar la calidad de la educación y orientar a aquellas escuelas que no cumplen con los estándares determinados. En ambos casos, se trata de órganos que, si bien ya están diseñando y/o implementando acciones formativas - el primero, a través de capacitaciones a escuelas que reciben subvención estatal, para incorporar acciones reparadoras a los manuales de convivencia (Pérez, 2013); y el segundo, a través del diseño de acciones de orientación para las escuelas que rinden por debajo de lo esperado (Huepe, 2013), su función fundamental es fiscalizar y evaluar, respectivamente. Por su parte el Mineduc, a través de la Unidad de Transversalidad, mantiene la Política Nacional de Convivencia Escolar (Mineduc, 2015), que entrega orientaciones para las escuelas, pero respeta su autonomía, la que es garantizada por la Ley General de Educación. En cambio, las leyes emanadas desde el poder legislativo, como tales, sí son obligatorias para las escuelas y restringen por tanto su autonomía y campo de acción.

Estamos, entonces, ante un escenario de política educativa ambivalente, en donde los sostenedores y las escuelas pueden traducir las políticas relacionadas con convivencia escolar — Ley de Violencia Escolar, Superintendencia Escolar, Agencia de Calidad de 
la Educación - siguiendo en el camino tanto una lógica penal como formativa; y apelando a la construcción autónoma de formas propias de gestión de la convivencia escolar, o siguiendo literalmente las leyes a las que están sujetas. Como señalan Cohen y Moffitt (2009), es justamente la ambivalencia de las políticas lo que genera tensiones en su implementación. A diferencia de un escenario de política educativa ambiguo, donde no hay claridad respecto de cómo actuar - pues el mensaje no es claro-, en un escenario ambivalente el mensaje es claro, pero es un doble mensaje que contiene dos polos o ejes discursivos contrapuestos (en este caso, uno punitivo y otro formativo). Esto genera tensiones en la implementación, pues los actores están sujetos a interpretaciones que pueden oscilar entre uno y otro eje discursivo (Cohen \& Moffit, 2009).

Los efectos de esta ambivalencia han sido reportados en algunos estudios acerca de la Ley de Subvención Escolar Preferencial (Ley SEP) (Mineduc, 2008), debido a que en ella subyace una tensión significativa al instalar una lógica de autonomía en la gestión escolar, junto con otra de amenaza y sanción, con impactos importantes para las culturas escolares (Ahumada, 2010; Assaél, Acuña, Contreras y Corbalán, 2014).

Chile, como muchos otros países, tiene desde hace décadas una prueba nacional de evaluación del rendimiento educativo (Simce). Sin embargo, hasta hace poco el Simce no tenía altas consecuencias para las escuelas, con excepción de la bonificación y premiación del sistema SNED como consecuencia positiva y del ranking público de colegios efectuado por los medios de prensa como potencial consecuencia negativa. En resumen, las consecuencias eran pocas. Sin embargo, con la Ley SEP (Mineduc, 2008) este escenario ha cambiado: las escuelas pueden bajar de categoría en su evaluación de gestión escolar, y se instala la amenaza de cierre derivada de la posibilidad de que el Mineduc retire el reconocimiento oficial. Si bien la Ley SEP no es obligatoria para los establecimientos que reciben subvención, la mayoría de las escuelas chilenas municipales y particulares subvencionadas están actualmente acogidas a la Ley SEP (Domínguez, 2014; Elacqua, 2012). La Ley SEP acentuó los mecanismos de control por resultados, consolidando la exigencia de 
104 TENSIONES Y NUDOS CRÍTICOS EN LA IMPLEMENTACIÓN DE LA(S) POLÍTICA(S) DE CONVIVENCIA ESCOLAR EN CHILE - V.López, L. Ramírez, R. Valdés, P. Ascorra y C. CarrascoAguilar

cumplimiento de estándares y la categorización de escuelas (GarcíaHuidobro y Bellei, 2006).

Esta lógica de responsabilización por resultados y financiamiento condicionado a ello fue heredada y fortalecida por el Sistema de Aseguramiento de la Calidad de la Educación, creado por la Ley de Aseguramiento de la Calidad de la Educación (Ley N 20.529, de aquí en adelante SAC). El SAC rediseñó y renombró la forma de categorización de los establecimientos escolares ${ }^{7}$.

En el marco del SAC, la Agencia de Calidad de la Educación Escolar agregó fuerza al escenario de altas consecuencias negativas (high-stakes testing). La Ley SAC estableció que dicha Agencia no solo velaría por evaluar los logros de aprendizaje y entregar orientaciones de mejora en el desempeño de los establecimientos educativos, incorporando para ello nuevos indicadores y estándares indicativos de desempeño como "el clima de convivencia escolar", sino que además ordenaría a las escuelas en las cuatro categorías definidas anteriormente según dicha evaluación. Luego de cuatro años, si un establecimiento se mantiene en la categoría de insuficiente, este será sancionado con la pérdida de su reconocimiento oficial, debiendo por consecuencia, cesar sus servicios.

Así, la Ley SAC ofreció dos miradas opuestas en materia de convivencia, proponiendo por un lado ordenar y clasificar escuelas, de modo de hacer competir a los establecimientos por el reconocimiento y valoración de la comunidad educativa; a la vez que estableció la convivencia escolar basada en valores de participación, trabajo colectivo, ejercicio de deberes y derechos y respeto a la diversidad como estándares indicativos de desempeño para los establecimientos y sus sostenedores.

Como señalan Cohen y Moffitt (2009), las políticas educativas son generalmente acompañadas de significados ambivalentes.

La Ley SEP definió las categorías de autónomas, emergentes y en recuperación; el SAC agregó una categoría adicional y las renombró como de desempeño alto, medio, medio-bajo e insuficiente, pero mantuvo las altas consecuencias bajo la amenaza de cierre a través del retiro de reconocimiento oficial. 
Siendo este generalmente el caso de las políticas de educación, mayor es su constatación para aquellas reformas que exigen muchos cambios a las escuelas, pero que ofrecen pocas guías respecto de cómo alcanzar los resultados esperados. Tal es el caso, en Estados Unidos, de la ley No Child Left Behind (NCLB); y en Chile, de la Ley SEP, de la LVE y, recientemente, de las mediciones no cognitivas de calidad de la educación que está implementando la recientemente creada Agencia Nacional de Calidad de la Educación. En todos estos casos, se responsabiliza a la escuela en la mejora de los aprendizajes de los estudiantes, se le exige contabilidad para dar cuenta de dicho avance, y se establecen de antemano las consecuencias negativas que ocurrirán si no cumple las metas. Sin embargo, como han señalado Raczynski et al. (2013) para el caso de la Ley SEP, una política de equidad requiere, al menos, establecer un equilibrio entre la presión por los resultados, con los apoyos y recursos pertinentes. De allí que nos proponemos comprender cómo ha sido traducida la política desde la perspectiva de los implementadores de nivel organizacional.

\section{Método}

\subsection{Participantes}

El estudio involucró a diez informantes clave (siete mujeres y tres hombres $)^{8}$ que participaban del diseño e implementación de políticas en convivencia escolar: tres provenían de la Agencia de Calidad, cinco del Ministerio de Educación y dos se desempeñaban en la Superintendencia de Educación. Siete de los participantes se encontraban en ejercicio profesional en las dependencias del nivel central, mientras que tres de ellos se desempeñaban en los niveles regionales y provinciales (Ministerio de Educación) o de macro zona (Superintendencia de Educación). Las personas entrevistadas fueron consideradas participantes clave por el nivel de relevancia que tenían en la toma de decisiones acerca de la implementación de las

Con el fin de resguardar la confidencialidad y anonimato de los participantes, solo se incluyen datos de caracterización de los participantes, el sexo y los años de desempeño laboral en la institución. 
políticas de convivencia escolar. Todos tenían cargos de dirección y/o de coordinación y contaban con suficiente trayectoria y antigüedad en los organismos donde trabajaban como para dar cuenta de las tensiones y nudos críticos. El promedio de años trabajando en cada institución fue de 6,1 (rango: 3 a 13 años).

\subsection{Técnica de producción}

Se aplicó la entrevista activo-reflexiva de Holstein y Gubrium (1995). Este tipo de entrevista — más ligada a la interacción que al contenido - se distancia de los modelos tradicionales de hacer entrevistas, pues asume la no neutralidad del entrevistador y, por el contrario, intenciona la participación activa del mismo a través de preguntas que movilizan conocimientos previos e incentivan la reflexión. Así, la entrevista consistió en una conversación de carácter activo en la que el entrevistador buscó que cada participante definiera y desarrollara las tensiones y/o contradicciones que él/ella visualizaba, en las actuales políticas de convivencia escolar, focalizándose en aquellas que él/ella veía u observaba desde el rol que le correspondía en calidad de agente diseñador, o bien de implementador de dichas políticas. De esta manera, todas las entrevistas comenzaron con las siguientes dos preguntas:

- ¿Cuáles son las tensiones y/o contradicciones que usted visualiza en las actuales políticas vinculadas a la convivencia escolar en el país, y cómo esto lo afecta en su actuar cotidiano?

- ¿Cuáles son los principales nudos críticos que usted visualiza en la implementación de la política en convivencia escolar?

Luego de ello, el entrevistador compartió con cada participante siete nudos críticos que el equipo de investigación había identificado en un estudio previo, tras realizar un análisis documental de las leyes y políticas de convivencia escolar (Ascorra, Carrasco, López y Morales, en revisión), se solicitaba a los participantes emitir su opinión respecto de dichos nudos críticos. Las entrevistas tuvieron una duración aproximada de 1 hora 30 minutos. 


\subsection{Procedimiento y análisis de datos}

Todos los participantes firmaron una carta de consentimiento informado. Las entrevistas se realizaron en las dependencias donde ellos trabajaban regularmente, resguardando las condiciones adecuadas de acústica e iluminación. En todas las entrevistas, se llevó a cabo un análisis de contenido categorial temático (Vázquez, 1994) con categorías emergentes. Se analizaron las entrevistas buscando los roles y funciones de los distintos organismos del SAC y las tensiones y nudos críticos que visualizaban los entrevistados. Los datos fueron analizados utilizando el programa de análisis cualitativo de datos Atlas ti versión 7.0.

El análisis de las entrevistas contempló los siguientes momentos: a) lectura de entrevistas en conjunto; b) codificación de citas según criterios de interés; c) reagrupamiento de citas en categorías y subcategorías; d) precisión de categorías en tópicos de estudio; y e) análisis e interpretación de resultados.

\section{Resultados}

Los resultados han sido organizados en tres tipos de tensiones identificadas por los participantes: conceptuales; metodológicas; y en la gestión de la convivencia escolar (ver Tabla 1). 
108 TENSIONES Y NUDOS CRÍTICOS EN LA IMPLEMENTACIÓN DE LA(S) POLÍTICA(S) DE CONVIVENCIA ESCOLAR EN CHILE - V.López, L. Ramírez, R. Valdés, P. Ascorra y C. CarrascoAguilar

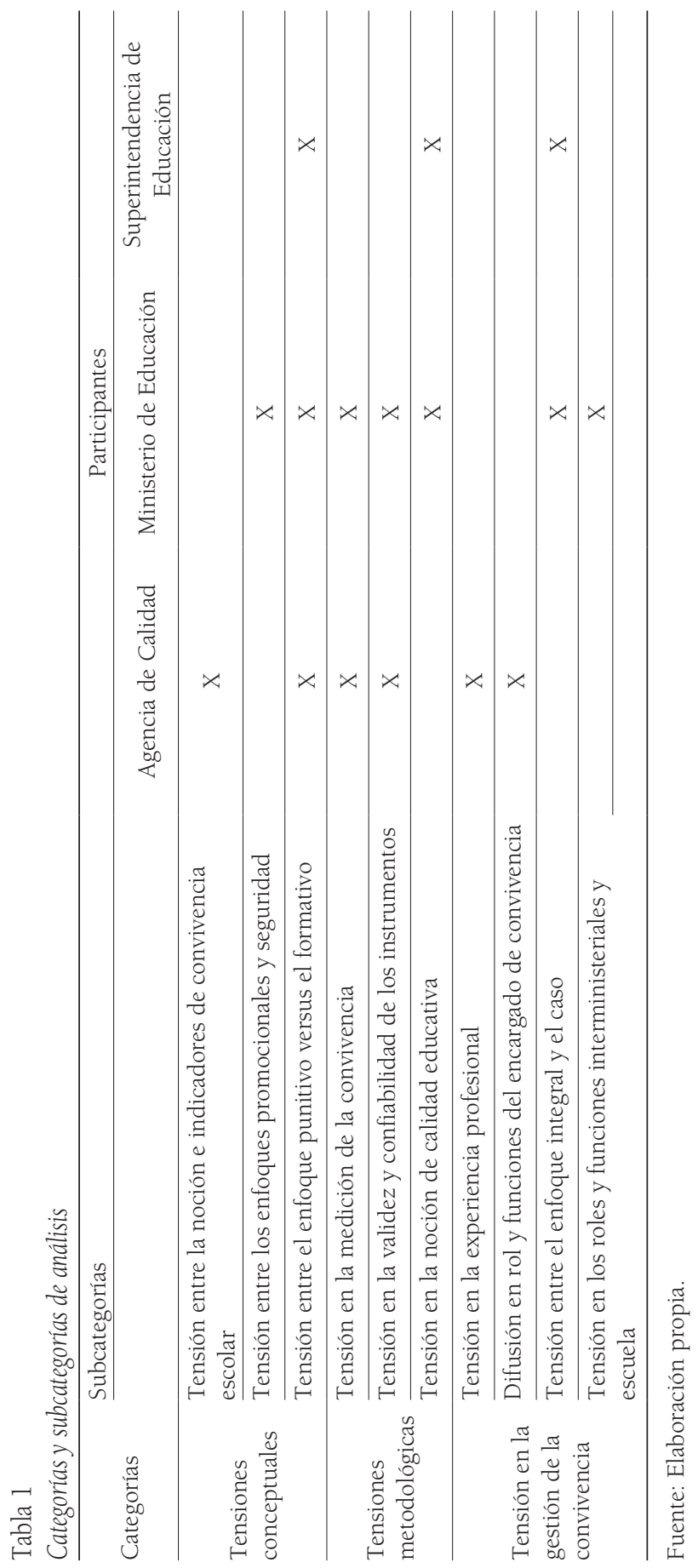




\subsection{Tensiones conceptuales}

Refiere a las dificultades respecto de lo que se entiende por convivencia escolar. Emergen tres subcategorías: tensión entre la noción y los indicadores de convivencia escolar; tensión entre el enfoque punitivo versus el formativo; y tensión entre los enfoques promocionales y seguridad.

5.1.1. Tensión entre el constructo y los indicadores de convivencia escolar

En relación con las tensiones entre la noción e indicadores de convivencia escolar, los participantes de la Agencia de Calidad refieren una tensión presente en cuanto a la falta de claridad respecto de lo que se entiende por convivencia escolar.

(...) mira, la verdad no te podría decir muy bien el tema, algo así como de la definición de clima de convivencia. Nosotros como que nos atenemos a esta definición previa que se hizo bibliográfica y teórica, que en el fondo definió por un lado la convivencia de los otros indicadores, pero por otro lado los estándares indicativos, y en ese sentido la verdad es que sí conversan, pero son [distintos] (Participante 8, Agencia de Calidad).

Esto tendría efectos tanto en los indicadores que se construyen para medir la convivencia escolar como en las prácticas educativas. Los participantes señalan que esa falta de claridad afecta el mensaje que la Agencia quiere dar en las escuelas, pues el rol de este organismo es orientar los procesos de mejoramiento cuando los establecimientos no alcanzan los resultados deseados en pruebas estandarizadas.

(...) y le están diciendo a las escuelas que tienen perfecta convivencia o mala convivencia, pero por otro lado se está usando otro constructo, y por otro lado hay unos planes de mejora que le dicen para lograr convivencia "Ud. haga esto otro". Finalmente, no, no estamos aportando. Entonces, eh, yo creo, que el mayor desafío y el problema político, es que es un tema candente, un tema que a todos preocupa y que esa es una oportunidad, pero que a todos ocupa, cada uno quiere tener su parcela, y cada uno 
110 TENSIONES Y NUDOS CRÍTICOS EN LA IMPLEMENTACIÓN DE LA(S) POLÍTICA(S) DE CONVIVENCIA ESCOLAR EN CHILE - V.López, L. Ramírez, R. Valdés, P. Ascorra y C. CarrascoAguilar

como que quiera avanzar. Y eso hace que las cosas se hagan inconexas y que cueste mandar un mensaje con toda la fuerza que se necesita (Participante 3, Agencia de Calidad).

\subsubsection{Tensión entre el enfoque punitivo versus el formativo}

Tanto los participantes de la Agencia de Calidad, del Mineduc y de la Superintendencia refirieron tensiones entre enfoques punitivos y formativos presentes en la política de convivencia escolar. Los entrevistados pertenecientes a la Agencia de Calidad aludieron a la tensión entre enfoques punitivos versus formativos que sostienen a la política de convivencia, que se materializa en el riesgo de cierre de los establecimientos si es que no cumplen los indicadores de calidad. Esta tensión se replica en implementaciones de tipo sancionadoras o formativas en los establecimientos escolares:

(...) de salir de esta rendición de cuenta tan castigadoras, sino que es una rendición de cuentas que busca ayudar, busca focalizar la mejora, como un Estado que orienta, que apoya, un Estado que entiende que al colegio hay que ayudarlo para que mejore y no exigirlo y castigarlo, eso me parece que está súper bien, ese cambio de paradigma me parece que hace falta, que permee más, que los instrumentos que nosotros ocupamos se pulan, que se terminen, terminemos de definirlos, terminemos de... no sé si algún día se terminará, pero en el fondo cabe ir mejorando más y más, ir aprendiendo más y más, y si yo veo algo crítico es el tema de cerrar los colegios a los cuatro años, creo que eso cuesta mucho sacarlo de los establecimientos ese fantasma, no sé, si ese es el camino va a generar que los colegios mejoren, me parece contradictorio a la base, pero es una cosa muy personal mía, pero sí me parece que los esfuerzos y la forma de entender el sistema de aseguramiento de la calidad, la forma en que el SAC está entendiendo la educación y está destinando sus recursos de forma correcta, me parece que hacia allá vamos, podrá ser poco, lo que tú quieras, pero la dirección es la correcta que es desde el apoyo (Participante 8, Agencia de Calidad). 
En este mismo sentido, para los representantes del Mineduc coexistirían perspectivas formativas y punitivas. Mientras que la primera se centra en otorgar apoyo a los establecimientos, el foco de la segunda es sancionar el incumplimiento. Esto se evidencia tanto en las políticas ministeriales, como en las prácticas relacionadas con el programa de convivencia escolar en los establecimientos escolares. Para los actores, esta tensión tiene consecuencias en las prácticas escolares pues, por un lado, las escuelas intentan transmitir valores asociados a una buena convivencia, pero por otro, se limitan al cumplimiento normativo de las políticas de convivencia escolar.

(...) está la lógica del control que se ha visto en las escuela y que se ha visto potenciada por las políticas ministeriales en términos de que cuando empieza a entrar en funcionamiento el Sistema de Aseguramiento de Calidad de la Educación se le da un bombo excesivo, por ejemplo, a la Superintendencia, que entonces ratifica en la escuela que lo que le importa al Ministerio es la sanción, y es el instrumento, da lo mismo cómo esté hecho, sino que dentro de esta lista de chequeo tenga en orden el reglamento, entonces cuando en la escuela está instalado eso y cuando el Ministerio lo está reforzando, es bastante difícil hacer ese cambio de mirada, a no ser por personajes individuales puede solucionar, porque finalmente es como el ying y el yang, lo bueno y lo malo, es que, que el mismo agente, por un lado te dice "te apoyo, te apoyo, te apoyo". Pero por otro lado si no lo logras, te castigo (Participante 4, Ministerio de Educación).

Por su parte, para los participantes de la Superintendencia, esta tensión entre enfoques punitivos y formativos afecta la forma de entender la convivencia escolar y sus correspondientes prácticas. Según los entrevistados, la complejidad en la manera en que los enfoques "bajan" a las escuelas afecta el trabajo de las mismas:

Claro, si en el fondo no está el foco formativo es difícil pensar en la medida reparatoria, es difícil para las escuelas que trabajan con un paradigma más punitivo reconocer, identificar la importancia de la reparación en el proceso formativo, por eso no está incluido. Yo creo que hay mucha deficiencia todavía en entender la disciplina 

Aguilar

formativa y que es necesaria, digamos, formar disciplina... no está, creo que hay escuelas que han ido avanzando en un cambio de paradigma, es difícil. Y los manuales [se refiere a los manuales de convivencia escolar que son obligatorios en todos los establecimientos escolares], yo diría la gran mayoría, sino casi todos, no tienen un real enfoque formativo, tienen algunas medidas como te decía en términos de oportunidades hasta la sanción, pero no están pensado en un manual para lograr que los estudiantes desarrollen las competencias que requieren para poder convivir con otros, para poder hacer su trabajo escolar (Participante 9, Superintendencia de Educación).

Según los entrevistados, esta tensión conceptual afecta tanto a los adultos como a los estudiantes en las escuelas puesto que, si la política pública es punitiva con las escuelas, estas aplicarán la misma lógica con los estudiantes. En ese sentido, se estaría traspasando un discurso que no necesariamente colabora con una mirada formativa y pedagógica acerca del trabajo en las escuelas:

Es cierto, la ley [se refiere a la LVE] es punitiva, suele ocurrir que los adultos del colegio, bueno los adultos no se están formando, por lo tanto entran en el mismo formato que entra uno que se pasa la luz roja, no se forma uno hasta que el carabinero no te pase el parte, por lo tanto se traspasa ahí mismo lo punitivo con la escuela cuando no cumple con ciertas normativas y eso muchas veces se traspasa de forma punitiva a los estudiantes, no se hace ahí como el giro, de que una institución también son adultos, se enmarca dentro de una ley que es punitiva, pero que ellos no pueden aplicar el mismo enfoque con los estudiantes (Participante 9, Superintendencia de Educación).

\subsection{Tensión entre los enfoques promocionales y de seguridad}

Los participantes del Ministerio de Educación identificaron tensiones en cuanto a los enfoques promocionales versus aquellos centrados en la seguridad. Esta tensión se genera entre enfoques centrados en el desarrollo humano y autocuidado de los estudiantes, junto con un enfoque de seguridad, asociado a la construcción de espacios 
seguros y de evitación de la violencia. Los entrevistados señalan que el concepto de convivencia debe ser aclarado desde la noción de prevención y desarrollo personal y no desde la seguridad escolar, pues este último es vinculante con la noción de violencia en la escuela:

No es que no haya evidencia, o no es que no haya información respecto de cómo evaluar la convivencia escolar, solamente si nos hubieran preguntado ya hubiéramos dicho que el concepto de seguridad en espacio seguro no lo hubiéramos puesto. Porque lo que nosotros incorporamos es el autocuidado y la prevención, cuando hablamos de seguridad humana, seguridad escolar, no hablamos y nos ha costado mucho hacer ese discurso, es otra de las cosas que, bueno, pero no la ley de convivencia, y nuevamente reforzamos el malentendido que convivencia es violencia, que convivencia es conflicto y que conflicto es algo que hay que evitar, o sea, doble error, porque convivencia no es violencia y si es violencia no hay un solo tipo de violencia (Participante 4, Ministerio de Educación).

\subsubsection{Tensión en la noción de calidad de la educación}

Los participantes de Mineduc precisan tensiones respecto de lo que se entiende por calidad de la educación. Conceptualmente centrada en aprendizajes y evaluación de los resultados, y la supremacía del dispositivo Simce por sobre otros instrumentos de calidad, los entrevistados señalan que hay un doble discurso respecto del concepto de calidad educativa. Por un lado, se promueve el aprendizaje integral y los otros indicadores de calidad; pero por otro, se pone el foco en la prueba Simce que mide rendimiento escolar en áreas disciplinarias del currículo y tiene importantes consecuencias para las escuelas.

Esperamos una comprensión de calidad, porque efectivamente ambivalente quizás puede ser la palabra. Yo creo que hay miradas hasta contradictorias. Calidad muy centrada en el resultado de pruebas, en la posición de Chile "en relación a", versus esta lógica de formación integral y les tiene que haber pasado a ustedes también, cuando me tocó compartir con varias escuelas con bajos resultados Simce, con bajo resultado académico en general, pero habían transitado a ser escuelas con impronta de ser los 

Aguilar

basureros de la comuna a tener un sentido de identidad, de mítica, de cohesión importante. Entonces, ¿qué es la calidad? Hoy día en el contexto de la Reforma, la calidad transita por esta lógica de formación integral, de inclusión educativa sin descuidar los resultados, por supuesto, pero entendiendo estos procesos. La escuela no lo tiene claro, entonces también coexisten. Te dicen [se refiere a las escuelas], no si nosotros tenemos, hemos mejorado la convivencia, los chiquillos están más contentos, pero sabe que no me metan más cachos, porque tengo que prepararlos para el Simce. Existe la constricción, que es la contradicción que le impone el sistema (Participante 4, Ministerio de Educación).

Los participantes que se desempeñan en la Superintendencia Escolar señalan las contradicciones inherentes a la conceptualización actual del sistema SAC respecto de la calidad de educación. Por un lado, reconocen que hay un llamado a una formación integral, pero al mismo tiempo, hay una evaluación de los aprendizajes en función de pruebas estandarizadas y con consideración principalmente curricular:

(...) sí, es que yo creo que la no sé, si es la política pública, pero la línea evaluativa de la, en Chile, cómo se ha evaluado la educación o la calidad de la educación está dada por dos instrumentos digamos, o principalmente dos instrumentos que es la PSU y el Simce, entonces en qué se centra eso, en los contenidos que definía el currículum, en fechas, no sé, en cálculos, en gramática, en comprensión lectora, que son temas súper fundamentales para la formación ciudadana de una persona, pero no es todo, o sea la Ley General dice de que la educación es un proceso integral que involucra distintas áreas, el área espiritual, el área emocional, el área relacional (Participante 10, Superintendencia de Educación).

\subsection{Tensiones metodológicas}

Los participantes también identifican tensiones metodológicas, referidas a cómo se evalúa la convivencia escolar en el marco del sistema SAC. Las subcategorías se refieren a la tensión en la medición del constructo y en la validez o confiabilidad de los instrumentos utilizados en los procesos evaluativos estandarizados. 


\subsubsection{Tensión en la medición de la convivencia}

En relación con la medición del constructo, los entrevistados de la Agencia de Calidad sostienen que existe una falta de acuerdo acerca de los instrumentos en cuanto a qué y cómo evaluar el constructo de convivencia escolar. El primer cuestionamiento se refiere a la efectividad de los procedimientos evaluativos. También se presentan reflexiones respecto de cómo comunicar estos resultados, pues los entrevistados aclaran que, al ser instrumentos de autorreporte, se sostienen en la percepción de las personas:

O sea teniendo en consideración que los cuestionarios, que el instrumento que se aplica para obtener los otros indicadores, se mide la percepción que tiene la gente o sea tú no estás midiendo efectivamente el clima, tú estás midiendo la percepción del clima, tú no estás midiendo la autoestima con un termómetro, estás midiendo la autoestima a través de lo que las personas dicen, entonces teniendo eso en consideración los instrumentos a nivel técnico yo entiendo que están súper validados con expertos nacionales e internacionales que se someten a constante revisión, lo que sí todavía está un poco pendiente y que a nosotros nos ha costado un poco es cómo comunicar los resultados, eso sí para nosotros ha resultado complicado, pero en términos de levantar los cuestionarios y etc. Por lo tanto, nosotros no estaríamos, viendo el puntaje del cuestionario, en base al constructo del cuestionario, sino que de otro. Entonces, existe yo creo que la mayor dificultad, es esto, la gran cantidad de constructos que existen, el cómo se está entendiendo la convivencia (Participante 8, Agencia de Calidad).

En cuanto a la validez y confiabilidad de los instrumentos, los participantes de la Agencia aclaran que el que los instrumentos sean autorreportados podrían sesgar la información recabada. Incluso sostienen que, en el contexto de la incorporación de los indicadores de desarrollo personal y social (IDPS) dentro de un sistema de rendición de cuentas con consecuencias para las escuelas, los estudiantes podrían verse entrenados en el ejercicio de responder los instrumentos de la Agencia de Calidad: 

Aguilar

Entonces, cuando hay preguntas como... claro hay preguntas, o sea respuestas correctas, pero es mucho más difícil que sea tan evidente, o sea las respuestas correctas. En cambio, en este tema, es mucho más evidente. Que ya los directores empiecen a decirle a los niños "no digan que no sé qué" o "sí digan que no sé cuánto". Yo creo que es la mayor debilidad y eso también juega en contra para el análisis con IRT [teoría de respuesta de ítem, IRT en su sigla en inglés], o sea para levantar los niveles de calidad también. Tenemos un súper gran desafío para definir estándares, qué es lo que vamos a entender por clima de convivencia adecuado, entonces ahí como que se nos viene una gran definición, conceptual también (Participante 7, Agencia de Calidad).

Los participantes del Mineduc comparten la visión de las tensiones en cuanto a medición del constructo de convivencia escolar y acerca de la validez y confiabilidad de los instrumentos. Desde el punto de vista de la medición, los entrevistados indican que no existe claridad respecto de cuáles son los indicadores de convivencia escolar, así como tampoco acerca de cómo se evalúan y su relación con el desempeño académico. Se duda de la rigurosidad del concepto de convivencia escolar y de la evaluación del mismo:

(...) pongámosla partiendo de la definición más simple que es la que está en el... la misma que tiene la ley [se refiere a la LVE] y la Superintendencia, ¿ya?, y luego de eso cómo hacemos para recoger información respecto a esto y otros indicadores, qué apoyos prestamos, a quiénes y cómo lo evaluamos a fin de año si efectivamente porque uno ve, eh, convivencia escolar que tienen tantas cosas, no, todos los talleres extracurriculares, además reforzamiento, además esto, ya, pero ustedes han evaluado eso, eso qué impacto tiene, en qué resultados institucionales, en eficiencia interna por ejemplo, en asistencia, en promoción, en repitencia o en aprendizaje no saben no saben y no saben, eh, y no saben pero hacen una cantidad de cosas que es así como un esfuerzo pero, por eso digo que es como palos de ciego, y un montón de cosas, y muchas cosas y cuántas de esas son las que realmente están impactando por ejemplo en esos otros indicadores (Participante 1, Ministerio de Educación). 
Por otro lado, para los participantes de Mineduc, no existe claridad respecto de la validación de los instrumentos que se utilizan para evaluar la convivencia escolar, pues desde su perspectiva, estos instrumentos responden más bien a un diseño administrativo que no necesariamente se condice con la realidad de los establecimientos escolares:

Entonces realmente no sé cuál es la validación de todos esos instrumentos, la validación finalmente administrativa, digo yo, legal, leyes detrás de un diseño de política más que una reflexión académica en base a literatura, la investigación, la misma realidad (Participante 2, Ministerio de Educación).

\subsection{Tensión en la gestión de la convivencia}

Los participantes refieren a tensiones en la gestión de la convivencia escolar. La mayor parte de las aprehensiones aluden a la necesidad de desarrollar nuevas competencias y experticia profesional para apoyar a las escuelas en el mejoramiento de la convivencia escolar.

\subsubsection{Tensión en la experiencia profesional acumulada}

Los participantes de la Agencia de Calidad señalan que esta nueva arquitectura de la educación chilena ha traído consigo nuevos roles $\mathrm{y}$ funciones $\mathrm{y}$, en ese sentido, se requieren nuevos profesionales y expertos. Sin embargo, y dada la reciente institucionalidad de la Agencia de Calidad, este organismo se encuentra aún en una etapa de clarificar los roles de sus profesionales y de volverlos expertos en los temas atingentes al organismo:

Sí, o sea nos hace falta formar una masa crítica de evaluadores con experiencia que puedan hacer bien la pega, o sea no estoy diciendo que la hagan mal hoy en día, pero en el fondo hemos tenido que ir aprendiendo todos, o sea ellos, muchos de ellos son profes o psicólogos, y que no están formados para este tipo de cosas en particular, y que tampoco había mucha gente que supiera mucho al respecto (Participante 8, Agencia de Calidad). 
118 TENSIONES Y NUDOS CRÍTICOS EN LA IMPLEMENTACIÓN DE LA(S) POLÍTICA(S) DE CONVIVENCIA ESCOLAR EN CHILE - V.López, L. Ramírez, R. Valdés, P. Ascorra y C. CarrascoAguilar

5.4.2. Ambigüedad en torno al rol y funciones del encargado de convivencia

En esa misma lógica, pero en relación con la escuela, los participantes señalan que el rol del encargado de convivencia escolar tampoco está claro. Aluden a una falta de lineamientos explícitos que definan las funciones de este profesional en el establecimiento:

No sé, yo creo que mientras no haya como horas disponibles para que el encargado se ocupe, y para que el sistema como también tú dices, o sea que se pueda elegir un encargado de manera de que sea orgánico, a las funciones que tiene que cumplir, en relación con las funciones que debe cumplir el colegio, como integralmente. No, no le veo mucha vuelta tampoco al encargado de convivencia escolar (Participante 7, Agencia de Calidad).

Los representantes del Mineduc mencionan que, en el espacio escolar, no hay claridad respecto del rol, funciones y tiempos destinados del encargado de convivencia, ni tampoco se cuenta con profesionales especializados en la temática:

La sobreexigencia en la escuela, pero particularmente al encargado de convivencia escolar y la escuela, y particularmente el encargado de convivencia escolar, cargo que se creó desde la Ley de Violencia es con la... debe tener conocimiento altamente especializado en convivencia escolar y se establece el requerimiento de formar en convivencia escolar, sin embargo, no queda claro de qué manera esta formación se lleva a cabo, ni cómo, qué tan especializado es el encargado de convivencia escolar. El efecto de esta tensión es la disminución de la capacidad de control y regulación del encargado de convivencia y de la escuela en materia, cierto, de la convivencia, del clima, y un alto nivel de estrés también asociado a una pérdida de control (Participante 1, Ministerio de Educación).

\subsubsection{Tensión entre un enfoque integral y la atención de casos}

En relación con las distintas miradas para la gestión de la convivencia, para los participantes del Mineduc, especialmente desde la voz de aquellos que se desempeñaban en los departamentos provinciales de educación como supervisores de educación, la temática de 
convivencia en las escuelas está centrada más bien en la atención de lo que las escuelas llaman "los casos de estudiantes problema", que en una mirada integral de convivencia. Su efecto es desviar la atención en la responsabilidad del establecimiento, atribuyéndosela al estudiante y a su familia:

Qué voy a supervisar aquí si está la escoba, o sea, hay que salir todo el mundo y yo a salir a buscar al niñito dónde está y el niño se había ido a la playa, entonces no sé, es algo terrible y tú, y tú dices, y ese tipo de cosas pasan y otras cosas pasan en un día... que es fácil meterte en esto y perder esta perspectiva de cuáles son, qué estamos entendiendo con esto, las mismas prácticas del modelo de gestión del Ministerio, o sea, nosotros le decimos no se salgan de eso de esas prácticas, eh, tomen una práctica llévenla al consejo, discutan, qué significa la atención a la diversidad, ya vamos a decir, ya, qué significa qué entiendes tú por atención a la diversidad, qué entiendo yo, ya, qué entendemos todos, vamos a definir esto, ya, esas cosas no están pasando. Entonces, hay una mirada que, en el fondo, que uno se da cuenta que es una mirada distinta, que es la que tenemos que cambiar, ya ahora, cuesta, porque como te digo la tendencia es ir al caso, ir a hablar de este apoderado, de esta alumna y que la hija fuma marihuana y que la mamá la deja que fume marihuana, esas cosas (Participante 1, Ministerio de Educación, nivel provincial).

En la misma línea que el organismo anterior, aparece la tensión referida a la forma de abarcar la convivencia en la escuela según un enfoque integral preventivo y/o promocional, o bien, a través de estrategias de atención caso a caso. Existe un cuestionamiento referido a la efectividad de las prácticas de gestión por parte de los entrevistados en el sentido de focalizar caso a caso. A lo anterior, los participantes de la Superintendencia agregaron que los protocolos en las escuelas están orientados principalmente hacia los alumnos, pero no en los adultos:

Esa es una parte del problema y lo otro, es que los colegios muchas veces focalizan sus energías en cumplir, con tener protocolos, o sea lo que la ley exige tampoco es tanto, es que dentro del manual 
120 TENSIONES Y NUDOS CRÍTICOS EN LA IMPLEMENTACIÓN DE LA(S) POLÍTICA(S) DE CONVIVENCIA ESCOLAR EN CHILE - V.López, L. Ramírez, R. Valdés, P. Ascorra y C. CarrascoAguilar

[de convivencia escolar] tengas un protocolo de actuación frente a las situaciones de agresión, no solo entre pares, también de adulto a niño, muchas veces tienen la de pares, pero no está la de adultoniño, o sea se mira la convivencia como de niño a niño entre ellos, eso es un problema. Tiene que tener un comité de convivencia, tiene que tener un encargado de convivencia, tienen que tener un plan de convivencia (Participante 9, Superintendencia de Educación).

5.4.4. Tensión en los roles y funciones interministeriales y entre ellos y las escuelas

En este punto, los entrevistados sostienen que se evidencia una tensión respecto de los roles y funciones que deben cumplir supervisores de Mineduc y Superintendencia. Concuerdan en que ello ha generado dificultades prácticas en la relación con los establecimientos escolares. Desde esta perspectiva, en el trabajo con las escuelas prima la desarticulación en la gestión de los distintos actores, existiendo distintos planes e instrumentos que definen prácticas diferentes de supervisión:

(...) absolutamente, mira y en esa relación SuperintendenciaMinisterio un factor re-relevante tiene que ver con cómo se configura, cómo se conformó la Superintendencia porque esto ya estaba por ley [se refiere a la Ley SAC], ya todos sabíamos que esto venía, pero fue bien traumática la implementación, porque por una parte le sacan funciones a la supervisión del Ministerio sin un proceso adecuado de inducción que permitiría a los supervisores reconvertirse, hubo muchas resistencias porque el supervisor, es genérico por supuesto, se sintió de repente como que le quitaron lo que él hacía, y dentro de la lógica más mediática se toma con supremacía el rol de la Superintendencia, entonces por supuesto ahí se impactó a las escuelas e impactó claramente cómo se llegaba al conjunto. Yo me acuerdo haber estado el año pasado, acá el año pasado, con la Superintendencia, con los supervisores, y cuando se habló de eso alguien dijo "es que en realidad a las escuelas les toca dos planes distintos" [se refiere al plan de mejoramiento escolar exigido por la Ley SEP y al plan de convivencia escolar exigido por la Superintendencia Escolar para dar cumplimiento a 
la LVE], las dos autoridades dijeron "sí, es un mismo plan", y uno sabe que en la práctica no es así como ellos lo dicen [pues tienen formatos distintos], entiendo muy claramente que son dos cosas distintas y de hecho si lo miras el instrumento o los documentos de apoyo, son muy distintos, si los instrumentos de apoyo son muy distintos uno interpreta "me están pidiendo trabajar el doble" (Participante 4, Ministerio de Educación).

\section{Conclusión}

El objetivo de esta investigación fue analizar las tensiones que identifican los actores que participan del diseño y ejecución de políticas educativas en convivencia escolar en Chile. Los resultados indicaron que las principales tensiones son conceptuales (qué es la convivencia escolar), metodológicas (cómo evaluarla) y procedimentales (cómo gestionar los apoyos para mejorar la convivencia escolar).

En relación con las tensiones conceptuales, todas las voces emanadas de los organismos entrevistados concuerdan en la presencia de una tensión semántica en la forma de entender la convivencia según los enfoques presentes en los documentos normativos, sean de la política pública o al interior de las escuelas. Por un lado, se hace presente un enfoque formativo — que invita a la reflexión y a la resolución pacífica de los conflictos-y, por otro, se explicita un enfoque punitivo (que busca la sanción y contiene una mirada castigadora de los problemas de convivencia). Ambos enfoques coexisten no solo en la política pública, sino también en la forma de gestionar la convivencia en la escuela. Los participantes de la Agencia de Calidad señalan, además, que esta falta de claridad afecta el mensaje que se quiere dar a los establecimientos y a todos los actores que participan del trabajo en convivencia escolar, ya sea en la forma de evaluar la convivencia como en la forma de mejorarla en las escuelas. Por su parte, los participantes que trabajan en el Mineduc agregan que, debido a esta imprecisión conceptual, también se ven afectados los procesos de promoción de la convivencia.

Esta tensión conceptual es concordante con los antecedentes teóricos derivados de investigaciones previas, los que dan cuenta 

Aguilar

de una concepción híbrida de convivencia escolar presente en las políticas de convivencia escolar en Chile (Carrasco et al., 2012; Magendzo et al., 2013). Se visualizan tensiones conceptuales asociadas a la coexistencia de distintas perspectivas y enfoques respecto de la convivencia escolar. Por una parte, los actores dan cuenta de un enfoque promocional, que se focaliza en el desarrollo integral de los estudiantes $\mathrm{y}$, por otra, un enfoque de seguridad centrado en la evitación de la violencia y en la regulación de comportamientos inapropiados, poniendo el énfasis en generar espacios seguros. A su vez, esta mirada se replica en las normativas y prácticas a nivel ministerial como en las escuelas, en las que se favorece en primera instancia un enfoque formativo en el que se busca acompañar al otro, ya sea a nivel de escuela o estudiante; y, al mismo tiempo, un enfoque punitivo, en el que prevalece la sanción ante el posible incumplimiento. Desde el punto de vista de la implementación, se observa cómo el carácter híbrido de la LVE (Carrasco et al., 2012; Magendzo et al., 2013) y, como hemos señalado en este artículo, del propio sistema de aseguramiento de la calidad, afecta directamente en una implementación también híbrida, tanto a nivel de la organización como de los individuos (Bowen $\&$ Zwi, 2005; Tabak et al., 2012), con un predominio al nivel organizacional (Bowen $\&$ Zwi, 2005) de un enfoque de tipo procedimental y punitivo.

En cuanto a las tensiones metodológicas, hay consenso en los discursos de los participantes de la Agencia de Calidad y del Mineduc en cuanto a las dificultades que visualizan en la medición del constructo de convivencia escolar. Se sostiene que no hay consensos explícitos acerca de qué se entiende por convivencia escolar y, por ende, de cómo evaluar ese constructo. Los instrumentos, al ser autorreportados, podrían incitar al entrenamiento de estudiantes en función de lo que se debe responder, ya que los instrumentos no están en la línea de evaluar las prácticas escolares de forma explícita (lo que hacen las escuelas). Estas tensiones despiertan las dudas en los entrevistados respecto del trabajo de la escuela y, sobre todo, del acompañamiento que puede hacer el SAC en los procesos de mejoramiento escolar. No encontramos tensiones metodológicas en la entrevista con los representantes de la Superintendencia de Educación. 
Sobre las tensiones en la gestión de la convivencia escolar, no hay tensiones que unifiquen los discursos entre los tres organismos investigados: los participantes de la Agencia de Calidad y del Mineduc concuerdan en la dispersión de roles y funciones en la figura del encargado de convivencia escolar de las escuelas, señalando que falta acuerdo acerca de la cantidad de horas de trabajo que se le pueden asignar, el perfil y su colaboración con otros actores de la escuela. También hubo señalamientos respecto de la necesidad de clarificar roles y funciones de los profesionales de la Agencia de Calidad que apoyan a los establecimientos escolares.

Naturalmente, los participantes de este estudio no lo hicieron como representantes oficiales de sus respectivos organismos, sino como sujetos implementadores de la(s) política(s) pública(s) en materia de convivencia y violencia escolar, estando ubicados en posiciones estratégicas en la actual implementación de dichas políticas. Tomando esto en consideración, y desde la perspectiva de las ciencias de implementación, los hallazgos de este estudio sugieren algunas debilidades a nivel de la organización, por cuanto las diversas políticas relacionadas con la convivencia escolar no habrían previsto la necesidad de "preparar el terreno" previamente a la implementación, a través de la estructuración de los perfiles y cargos, y de la formación y desarrollo de capacidades profesionales en los distintos niveles del sistema. Una posibilidad podría haber sido planificar la implementación de manera gradual y progresiva, tal como ha ocurrido recientemente con otras leyes educativas de gran alcance (por ejemplo, Ley de Inclusión No 20.845, del año 2015). Por el contrario, en este caso las políticas de convivencia escolar "irrumpieron" en el escenario escolar desde diversas fuentes legales, sin que el sistema - y por tanto las organizaciones y sus personas - se haya preparado organizacionalmente para ello (Bowen \& Zwi, 2005).

Por otra parte, las tensiones presentes en la gestión de la convivencia escolar guardan relación con el lugar que ocupa la temática dentro de la noción de calidad de la educación. Desde la perspectiva de los implementadores de la política educativa, el sistema privilegia los resultados de aprendizaje medidos a través del 

Aguilar

dispositivo Simce, por lo que el resto de los temas educativos se definen más bien por oposición; es decir, se encasillan dentro de lo que no aborda el Simce. De esta manera, la convivencia escolar sería definida como "lo que no es Simce". El efecto de esto es hacer prevalecer una visión fragmentada respecto de lo que se entiende por educación y convivencia escolar, que a su vez privilegia prácticas desarticuladas, que se materializan en distintos programas y, a su vez, en la difusión de funciones y roles que se deben cumplir. Por otra parte, señalan que la política promueve una formación integral con foco en diferentes dimensiones (convivencia, ciudadanía, etc.), pero en la práctica lo que cobra relevancia son los resultados en pruebas estandarizadas, las cuales fijan su atención en contenidos clave del currículo escolar (gramática, cálculo, etc.). Esto despierta otra confusión —o distracción— en la forma de trabajar de las escuelas, puesto que están presionadas para rendir en todas las áreas de la formación integral, pero son evaluadas y juzgadas por su rendimiento en áreas curriculares disciplinares en la prueba Simce.

Por último, tanto los participantes de la Superintendencia de Educación como los del Mineduc sostienen que las escuelas tienen desafíos en la gestión de la convivencia desde el punto de vista de "la mirada" o el enfoque del trabajo con las personas, pues aclaran que muchas veces los establecimientos focalizan sus prácticas en casos puntuales de problemas de comportamiento, por lo general por parte de los alumnos, y no en torno a una visión más global o integral sobre mejoramiento de la convivencia, en que no se incluya solo a los alumnos, sino a los profesionales y adultos.

Desde el marco conceptual de las ciencias de la implementación (Bowen \& Zwi, 2005; Tabak et al., 2012; Thomas et al., 2016), en este estudio fue posible analizar tensiones clave en la implementación de las políticas de convivencia escolar. La vía para probar políticas informadas a través de informantes clave involucrados en el diseño y/o ejecución de las políticas de convivencia escolar desde la implementación de la Ley SAC permite ilustrar las complejidades en la ejecución de políticas de mejoramiento de la convivencia escolar, insertas en un contexto mayor de una política de aseguramiento de la calidad basada en la responsabilización por desempeño (Falabella y 
De la Vega, 2016). Los hallazgos advierten de la necesidad de discutir los supuestos sobre los cuales se basa la arquitectura construida por la Ley SAC, particularmente, la prueba estandarizada con altas consecuencias a través del ordenamiento de escuelas. Desde una perspectiva social ecológica, los hallazgos también arrojan luces respecto de un ambiente de política progresivamente punitivo (Cohen \& Moffitt, 2009) que enmarca, a nivel de la organización y del sistema, los esfuerzos individuales por mejorar la convivencia escolar en las escuelas chilenas.

Por lo tanto, si se desea maximizar el éxito de los programas o políticas públicas que se deseen implementar en materia de convivencia escolar (Smith \& Polaha, 2017; Tabak et al., 2012), sostenemos que es necesario considerar de manera rigurosa las voces y experiencia de quienes han estado encargados de implementar la(s) política(s) de convivencia escolar durante los últimos años, con el objetivo de mejorar, a nivel de sistema y de organización, la calidad, eficiencia en tiempo y recursos y efectividad de los esfuerzos llevados a cabo a nivel individual (Eccles \& Mittman, 2006; Smith \& Polaha, 2017). Específicamente, los resultados de este estudio sugieren que es necesario, en primer lugar, precisar con mayor claridad la definición conceptual del constructo de convivencia escolar y su operacionalización orientada a la evaluación de las dimensiones y subdimensiones de dicho constructo. En segundo lugar, que es necesario adoptar, a nivel de la organización (Bowen \& Zwi, 2005), un enfoque de formación de capacidades profesionales (human capacity building) de los operadores de la política educativa, tanto a nivel central como regional, provincial y al interior de los establecimientos escolares. Un elemento clave parece ser la formación de capacidades de gestión y formación del encargado de convivencia escolar (Valenzuela, Ahumada, Rubilar, López y Urbina, 2018). En tercer lugar, y a nivel de sistema (Bowen $\&$ Zwi, 2005), los hallazgos de este estudio sugieren que es necesario abordar el ambiente de política punitivo (Cohen \& Moffitt, 2009) y centrado en el cumplimiento de la normativa que el sistema está haciendo prevalecer, por sobre un enfoque formativo, preventivo y promocional de la convivencia escolar (Ascorra et al., 2015; Ascorra, López, Carrasco, Pizarro, Cuadros y Núñez, 2018). 
126 TENSIONES Y NUDOS CRÍTICOS EN LA IMPLEMENTACIÓN DE LA(S) POLÍTICA(S) DE CONVIVENCIA ESCOLAR EN CHILE - V.López, L. Ramírez, R. Valdés, P. Ascorra y C. CarrascoAguilar

Esperamos que estos resultados y su discusión sirvan de insumo para las modificaciones a la propia Ley SAC definidas para el año 2019, y aporte al diseño, rediseño e implementación eficiente y efectiva (Eccles \& Mittman, 2006; Smith \& Polaha, 2017) de políticas públicas para el mejoramiento de la convivencia escolar y de la calidad de la educación.

\section{Referencias}

Astete, M. y Vaccari, P. (2017). Políticas públicas y subjetividades: lógicas en disputa en la implementación de programas sociales en la comuna de Lota, Chile. Psicoperspectivas. Individuo y Sociedad, 16(1), 31-41. https://doi.org/10.5027/psicoperspectivas-voll6-issuel-fulltext-880

Ahumada, L. (2010). Liderazgo distribuido y aprendizaje organizacional: tensiones y contradicciones de la Ley de Subvención Escolar Preferencial en un contexto rural. Psicoperspectivas. Individuo y Sociedad, 9(1), 111-123. https://doi.org/10.5027/psicoperspectivas-Vol9-Issuel-fulltext-99

Ascorra, P., López, V., Bilbao, M., Carrasco, C., y Morales, M. (2015). La convivencia escolar como práctica social: de lo individual a lo social, de lo reactivo y punitivo a lo formativo, del déficit a los recursos, de lo autoritario a lo participativo. En V. López, H. Díaz, y C. Carrasco (Eds.), Nosotros sí podemos: análisis de las buenas prácticas en convivencia escolar (pp. 117-141). Santiago de Chile: Centro de Investigación Avanzada en Educación (CIAE).

Ascorra, P., López, V., Carrasco, C., Pizarro, I., Cuadros, O., y Núñez, C. (2018). Significados atribuidos a la convivencia escolar por equipos directivos, docentes y otros profesionales de escuelas chilenas. Psykhe, 27(1), 1-12. http://dx.doi.org/10.7764/psykhe.27.1.1214

Ascorra, P., Carrasco, C., López, V. y Morales, M. (en revisión). La política pública sobre convivencia escolar en Chile: Análisis de sus contradicciones y nudos críticos. Enviado a revisión.

Assaél, J., Acuña, F., Contreras, P., y Corbalán, F. (2014). Transformaciones en la cultura escolar en el marco de la implementación de políticas de accountability en Chile: un estudio etnográfico en dos escuelas clasificadas en recuperación. Estudios Pedagógicos (Valdivia), 40(2), 07 26. https://dx.doi.org/10.4067/S0718-07052014000300001

Bowen, S. \& Zwi, A. B. (2005). Pathways to "evidence-informed" policy and practice: A framework for action. PLoS Medicine, 2(7), el66. https://doi.org/10.1371/journal.pmed.0020166 
Carrasco, C., López, V., y Estay, C. (2012). Análisis crítico de la Ley de Violencia Escolar de Chile. Psicoperspectivas. Individuo y Sociedad, 11(2), 31-55. http://dx.doi.org/10.5027/psicoperspectivas-Voll1-Issue2-fulltext-228

Cohen, D. K. \& Moffitt, S. L. (2009). The ordeal of equality: Did federal regulation fix the schools? Cambridge, MA: Harvard University Press.

Comisión de Educación, Deportes y Recreación. (2011). Informe de la Comisión de Educación, Deportes y Recreación, recaído en el proyecto de ley originado en moción que renueva la vigencia de la Ley $N^{\circ} 19.648$ de 1999, sobre acceso a la titularidad de los docentes a contrata en los establecimientos públicos subvencionados (Boletín 8784-04). Recuperado de https://www.camara. $\mathrm{cl} /$ pley/pdfpley.aspx?prmID=16008\&prmTIPO=INFORMEPLEY

Cornejo, R. y Reyes, L. (2008). La cuestión docente. Chile: experiencias organizacionales y acción colectiva de profesores. Santiago de Chile: FLAPE.

Domínguez, P. (2014). El impacto de la subvención escolar preferencial (SEP) en la composición socioeconómica de las escuelas: un análisis preliminar (Documento de trabajo). Recuperado de https://www.espaciopublico. cl/dominguez-patricio-el-impacto-de-la-subvencion-escolarpreferencial-sep-en-la-composicion-socioeconomica-de-las-escuelasdocumento-de-referencia-n15-septiembre-de-2014-48-paginas/

Eccles, M. \& Mittman, B. (2006). Welcome to implementation science. Implementation Science, 1(1), 1-3. https://doi.org/10.1186/1748-5908-1-1

Elacqua, G. (2012). The impact of school choice and public policy on segregation: Evidence from Chile. International Journal of Educational Development, 32(3), 444-453.

https://doi.org/10.1016/j.ijedudev.2011.08.003

Falabella, A. y De la Vega, L. (2016). Políticas de responsabilización por desempeño escolar: un debate a partir de la literatura internacional y el caso chileno. Estudios Pedagógicos, 42(2), 395-413. https://doi.org/10.4067/s0718-07052016000200023

Foy, R., Sales, A., Wensing, M., Aarons, G. A., Flottorp, S., Kent, B., ... Wilson, P. (2015). Implementation science: A reappraisal of our journal mission and scope. Implementation Science: IS, 10(51). https://doi.org/10.1186/s13012-015-0240-2

García-Huidobro, J. E y Bellei, C. (2006). ¿Remedio para la inequidad? La subvención escolar preferencial. Revista Mensaje. Recuperado de http://www.facso.uchile.cl/psicologia/epe/_documentos/GT_cultura_ escolar_politica_educativa/recursos\%20bibliograficos/articulos\%20 sep/garciahuidobroybellei(2006)leysepunremedioparalainequidad.pdf 
128 TENSIONES Y NUDOS CRÍTICOS EN LA IMPLEMENTACIÓN DE LA(S) POLÍTICA(S) DE CONVIVENCIA ESCOLAR EN CHILE - V.López, L. Ramírez, R. Valdés, P. Ascorra y C. CarrascoAguilar

Hevia, R. (2010). Apuntes para promover una ley sobre convivencia y violencia escolar. En P. Calderón (Ed.), Violencia escolar: una mirada desde la investigación y los actores educativos (pp. 45-54). Valparaíso: Universidad de Playa Ancha-Biblioteca del Congreso Nacional.

Huepe, M. (junio de 2013). Políticas públicas comparadas en abordaje de violencia en las escuelas. Trabajo presentado en el Congreso Iberoamericano de Violencia Escolar "Conversar sobre la cultura escolar para construir convivencia", Santiago, Chile.

Holstein, J. A. \& Gubrium, J. F. (1995). The active interview. Thousand Oaks, CA: Sage.

Leiras, M. (2007). Estudio de caso: la relación entre el Ministerio de Educación y el Colegio de Profesores de Chile, 1990-2006. Buenos Aires: Centro de Estudios de Política Educativa.

Magendzo, A., Toledo, M. I., y Gutiérrez, V. (2013). Descripción y análisis de la Ley sobre Violencia Escolar ( $\mathrm{N}^{\circ}$ 20.536): dos paradigmas antagónicos. Estudios Pedagógicos, 39(1), 377-391. https://dx.doi.org/10.4067/S0718-07052013000100022

Ministerio de Educación de Chile, Mineduc. (2008). Ley de Subvención Escolar Preferencial. Santiago de Chile: Autor.

Ministerio de Educación de Chile, Mineduc. (2011). Política de Convivencia Escolar. Santiago de Chile: Autor.

Ministerio de Educación de Chile, Mineduc. (2015). Política Nacional de Convivencia Escolar 2015/2018. Santiago de Chile: Autor.

Pérez, M. (junio de 2013). Políticas públicas comparadas en abordaje de violencia en las escuelas. Trabajo presentado en el Congreso Iberoamericano de Violencia Escolar, "Conversar sobre la cultura escolar para construir convivencia", Santiago, Chile.

Rabin, B. A., Purcell, P., Naveed, S., Moser, R. P., Henton, M. D., Proctor, E. K., ... \& Glasgow, R. E. (2012). Advancing the application, quality and harmonization of implementation science measures. Implementation Science: IS, 7(119). http://doi.org/10.1186/1748-5908-7-119

Raczynski, D., Muñoz, G., Weinstein, J., y Pascual, J. (2013). Subvención Escolar Preferencial (SEP) en Chile: un intento por equilibrar la macro y micropolítica escolar. Revista Iberoamericana sobre Calidad, Eficacia y Cambio en Educación, 11(2), 165-193.

Smith, J. D. \& Polaha, J. (2017). Using implementation science to guide the integration of evidence-based family interventions into primary care. Families, Systems, \& Health, 35(2), 125-135.

http://dx.doi.org/10.1037/fsh0000252 
Soto Guzmán, V. (2006). Profesionalización docente: desde la visibilidad a la invisibilidad. Revista Docencia, 30. Recuperado de http://www. revistadocencia.cl/new/wp-content/pdf/20100726081556.pdf

Tabak, R. G., Khoong, E. C., Chambers, D. A., \& Brownson, R. C. (2012). Bridging research and practice: Models for dissemination and implementation research. American Journal of Preventive Medicine, 43(3), 337-350. https://doi.org/10.1016/j.amepre.2012.05.024

Tello, C. (2013). Notas reflexivas y descriptivas sobre el enfoque de las epistemologías de la política educativa. Conjectura, 18, 48-62. Recuperado de http://www.ucs.br/etc/revistas/index.php/conjectura/ article/viewFile/2101/pdf_187

Tenorio, S. (2011). Formación inicial docente y necesidades educativas especiales. Estudios Pedagógicos (Valdivia), 37(2), 249-265. https:// dx.doi.org/10.4067/S0718-07052011000200015

Thomas, D., Berry, A., Djuricich, A., Kitto, S., Kreutzer, K., Van Hoof, T., ... $\&$ Davis, D. (2016). What is implementation science and what forces are driving a change in medical education? American Journal of Medical Quality, 32(4), 438-444. https://doi.org/10.1177/1062860616662523

Valenzuela, J., Ahumada, I., Rubilar, A., López, V., y Urbina, C. (2018). El encargado de convivencia escolar en Chile: hacia la comprensión de su identidad laboral. Revista de Psicología, 36(1), 189-216. https://doi.org/10.18800/psico.201801.007

Vázquez, F. (1994). Análisis de contenido categorial: el análisis temático. Barcelona: Universidad Autónoma de Barcelona.

Recibido: 20/09/2017

Aceptado: 22/05/2018 